\title{
Galectin-3 Derived from HucMSC Exosomes Promoted Myocardial Fibroblast-to-Myofibroblast Differentiation Associated with $\beta$-catenin Upregulation
}

\author{
Qinyu Guo ${ }^{1, *}$, Yuanyuan Zhao,* ${ }^{1, *}$ Jiejie $\mathrm{Li}^{1}$, Chao Huang ${ }^{1}$, Hua Wang ${ }^{2}$, \\ Xiangdong Zhao ${ }^{3}$, Mei Wang', Wei Zhu ${ }^{1}$ \\ ${ }^{1}$ School of Medicine, Fiangsu University, Zhenjiang, China \\ ${ }^{2}$ Department of Obstetrics, Affiliated Hospital of Fiangsu University, Zhenïang, China \\ ${ }^{3}$ Department of Clinical Laboratory, Zhenjiang Provincial Blood Center, Zhenjiang, China
}

Background and Objectives: Galectin-3 promotes fibroblast-to-myofibroblast differentiation and facilitates injury repair. Previous studies have shown that exosomes derived from human umbilical cord mesenchymal stem cells (hucMSC-ex) promote the differentiation of myocardial fibroblasts into myofibroblasts under inflammatory environment. Whether hucMSC-ex derived Galectin-3 (hucMSC-ex-Galectin-3) plays an important role in fibroblast-to-myofibroblast differentiation is the focus of this study.

Methods and Results: Galectin-3 was knocked-down by siRNA in hucMSCs, and then exosomes were extracted. Fibroblasts were treated with LPS, LPS + hucMSC-ex, LPS + negative control-siRNA-ex (NC-ex), or LPS + Galectin-3-siRNA-ex (si-ex) in vitro. The coronary artery of the left anterior descending (LAD) branch was permanently ligated, followed by intramyocardial injection with phosphate buffered saline(PBS), hucMSC-ex, hucMSC-NC-ex, or hucMSC-si-ex in vivo. Western blot, RT-PCR, and immunohistochemistry were used to detect the expression of markers related to fibroblast-to-myofibroblast differentiation and inflammatory factors. Migration and contraction functions of fibroblasts were evaluated using Transwell migration and collagen contraction assays, respectively. $\beta$-catenin expression was detected by western blot and immunofluorescence. The results showed that hucMSC-ex increased the protein expression of myofibroblast markers, anti-inflammatory factors, and $\beta$-catenin. HucMSC-ex also reduced the migration and promoted the contractility of fibroblasts. However, hucMSC-si-ex did not show these activities.

Conclusions: HucMSC-ex-Galectin-3 promoted the differentiation of cardiac fibroblasts into myofibroblasts in an inflammatory environment, which was associated with increased $\beta$-catenin levels.

Keywords: Mesenchymal stem cell, Galectin-3, Cardiac fibroblasts, Myofibroblasts

Received: November 11, 2020, Revised: February 1, 2021,

Accepted: February 3, 2021, Published online: April 30, 2021

Correspondence to Wei Zhu

School of Medicine, Jiangsu University, 301 Xuefu Road, Zhenjiang, Jiangsu 212013, China

Tel: +86-511-85033768, Fax: +86-511-85038483

E-mail: zhuwei@ujs.edu.cn

*These authors contributed equally to this work

(a) This is an open-access article distributed under the terms of the Creative Commons Attribution Non-Commercial License (http://creativecommons.org/ licenses/by-nc/4.0/), which permits unrestricted non-commercial use, distribution, and reproduction in any medium, provided the original work is properly cited.

Copyright (c) 2021 by the Korean Society for Stem Cell Research

\section{Introduction}

Myocardial infarction results in massive myocardial cell death and remodeling. Myocardial fibroblasts are one of the most abundant non-cardiomyocyte cells types in the myocardium and are considered effector cells of cardiac remodeling (1). To maintain myocardial structure and function in the early period after myocardial infarction, myocardial fibroblasts differentiate into myofibroblasts (2). Mesenchymal stem cells have a protective effect on myocardial injury. It has been shown that after intra- 
venously injecting mesenchymal stem cells, they specifically migrate to the site of myocardial injury to reduce inflammatory responses (3). Bone marrow mesenchymal stem cell exosomes can improve myocardial injury by attenuating inflammatory responses (4). Our previous studies showed that exosomes derived from human umbilical cord mesenchymal stem cells can improve cardiac function after acute myocardial infarction and promote the differentiation of myocardial fibroblasts into myofibroblasts under inflammatory environment, which is cardioprotective (5). Exosomes contain cytokines, proteins, and nucleic acids, which are then selectively transported to other cells (6). Which exosome proteins play key cardioprotective roles deserves further investigation.

Galectin-3 is a $30 \mathrm{kDa}$ protein with no enzymatic activity that is a member of the $\beta$-galactosid-specific lectin family (7). Galectin-3 has a variety of biological functions and plays a number of important regulatory roles in inflammation, immunity, and cancer (8). Recent evidence suggests that Galectin-3 is pivotal in the development of fibrosis in the heart, blood vessels, kidneys, liver, and lungs $(9,10)$. Additionally, Galectin-3 is critical for maintaining ventricular geometry and function in the first few days after myocardial infarction (11). A previous study showed that secretion of Galectin-3 by macrophages could promote the differentiation of cardiac fibroblasts into myofibroblasts after myocardial injury (12). We detected high Galectin-3 expression in hucMSC-ex, and in this study, we investigated its role in the differentiation of cardiac fibroblasts into myofibroblasts.

Fibrosis and the progression of renal, lung, liver, heart, and skin cancer are closely related to abnormal activation of the Wnt signaling pathway (13). The Wnt/ $\beta$-catenin signaling pathway can affect fibroblast function, fibrosis, and tissue repair (14). $\beta$-catenin is a key molecule in the canonical Wnt signaling pathway. Studies have shown that Galectin-3 is a new binding partner of $\beta$-catenin. Galectin-3 can bind $\beta$-catenin and directly regulate target gene transcription into the nucleus (15). Galectin-3 is considered a critical protein for modulating Wnt/ $\beta$-catenin signaling. In this paper, we show that Galectin-3 derived from hucMSC-ex promotes fibroblast-to-myofibroblast differentiation, which was associated with increased $\beta$ -catenin expression and nuclear translocation.

\section{Materials and Methods}

\section{Animals}

The animals used in this study included newborn Sprague-Dawley (SD) rats (1 3-days-old) and adult male
SD rats $(180 \sim 220 \mathrm{~g})$. All animal experiments were approved by the Animal Experiment Center of Jiangsu University in Zhenjiang, Jiangsu Province, China, and were conducted in accordance with the Guidelines for the Care and Use of Experimental Animals.

\section{Cell culture}

HucMSCs were isolated and cultured from umbilical cords of healthy newborn infants according to previously published protocols (16). In this study, hucMSCs were sourced from Affiliated Hospital of Jiangsu University (Jiangsu, China), and all subjects provided informed consent. HucMSCs were cultured in Minimum Essential Medium Alpha ( $\alpha$-MEM; Gibco, Grand Island, NY, USA) with $10 \%$ fetal bovine serum (FBS; Biological Industries, Beit HaEmek, Israel) at $37^{\circ} \mathrm{C}$ in $5 \% \mathrm{CO}_{2}$. Primary myocardial fibroblasts were isolated from the hearts of $1 \sim$ 3 -days-old SD rats. The ventricles were cut into pieces in HBSS buffer, digested with trypsin overnight at $4{ }^{\circ} \mathrm{C}$, and then with trypsin inhibitor and type II collagenase (Worthington, Lakewood, NJ USA) at $37^{\circ} \mathrm{C}$. The digested cells were cultured in high-glucose Dulbecco's modified Eagle's medium (H-DMEM; Gibco) containing 10\% FBS at $37^{\circ} \mathrm{C}$ with $5 \% \mathrm{CO}_{2}$ for $90 \mathrm{~min}$. After removing non-adherent cells, the adherent cells (myocardial fibroblasts) continued to be cultured in the same condition.

\section{Nanoparticle tracking analysis (NTA)}

HucMSC-ex were diluted with PBS filtered by a $0.22 \mu \mathrm{m}$ filter to achieve the optimal concentration detected by Nanosight nanoparticle analyzer. HucMSC-ex diluents were pushed into the sample room slowly. Ultimately, automatic video capture and data analysis were performed.

\section{HucMSC-ex tracker}

HucMSC-ex were labeled according to the instructions of CM-Dil (Invitrogen, Carlsbad, CA, USA). HucMSC-ex were resuspended in $1 \mathrm{ml}$ of PBS, and then CM-Dil dye was added at a ratio of $5 \mu \mathrm{l} / \mathrm{ml}$ and incubated at $37^{\circ} \mathrm{C}$ for $30 \mathrm{~min}$ in the dark. Then, the mixture was centrifuged in a $100-\mathrm{kDa}$ molecular weight cut-off (MWCO) ultrafiltration centrifuge tube at $1,000 \times \mathrm{g}$ for $30 \mathrm{~min}$ and washed with PBS to remove unbound dye. The control group underwent the same treatment with PBS replacing hucMSC-ex. Finally, hucMSC-ex labeled with CM-Dil or control CM-Dil were added to cardiac fibroblasts and incubated at $37^{\circ} \mathrm{C}$ for $12 \mathrm{~h}$. The cardiac fibroblasts were washed three times with PBS, fixed with 4\% paraformaldehyde for $20 \mathrm{~min}$, and then washed three times with PBS. Afterwards, nuclei were stained with Hoechst 
(Sigma-Aldrich, St. Louis, MO, USA) for $10 \mathrm{~min}$, and then the cells were imaged with a fluorescence microscope (OLYMPUS DP73, Tokyo, Japan).

\section{Galectin-3 siRNA transfection}

Three siRNA constructs targeting human Galectin-3 (siRNA1: 5'-GAACAACAGGAGAGTCATT-3', siRNA2: 5'CGGTGAAGCCCAATGCAAA-3', and siRNA3: 5'-TGCT TTAGATTTCCAAAGA-3') and a negative control (NC) construct were constructed by RIBOBIO (Guangzhou, China). When the density of hucMSCs reached $60 \%$ $70 \%$, they were transfected with NC or Galectin-3 siRNAs using Lipofectamine 2000 (Invitrogen).

\section{Exosome extraction}

When the density of hucMSCs reached $70 \% \sim 80 \%$, the cells were cultured in $\alpha$-MEM supplemented with $10 \%$ exosome-free FBS for $48 \mathrm{~h}$. The supernatant was collected and centrifuged at $300 \times \mathrm{g}$ for $20 \mathrm{~min}, 800 \times \mathrm{g}$ for $20 \mathrm{~min}$, $2,000 \times \mathrm{g}$ for $20 \mathrm{~min}$, and $10,000 \times \mathrm{g}$ for $30 \mathrm{~min}$ (all at $4^{\circ} \mathrm{C}$ ) to remove cells and debris. The supernatant was then concentrated with MWCO ultrafiltration centrifuge tubes at $2,000 \times \mathrm{g}$ for $30 \mathrm{~min}$, mixed with exoquick-TC exosomes separation reagent (System Biosciences, Palo Alto, CA, USA) overnight at $4^{\circ} \mathrm{C}$. The next day, the mixture was centrifuged at $12,000 \times \mathrm{g}$ for $30 \mathrm{~min}$ at $4^{\circ} \mathrm{C}$. Finally, extracted exosomes were resuspended in the appropriate volume of PBS and stored at $-80^{\circ} \mathrm{C}$. The protein concentration of exosomes was measured with the BCA kit.

\section{Acute myocardial infarction (AMI) model}

Male SD rats were randomly divided into five groups: sham, $\mathrm{AMI}+\mathrm{PBS}, \mathrm{AMI}+\mathrm{ex}, \mathrm{AMI}+\mathrm{NC}-\mathrm{ex}$, and $\mathrm{AMI}+$ si-ex. Except for the sham operation group, all animals received permanent coronary artery ligation according to previously reported methods (13). Next, PBS, hucMSC-ex, hucMSC-NC-ex, or hucMSC-si-ex (400 $\mu \mathrm{g})$ were immediately injected into the area around the ligation. After 48 $h$, the rats were sacrificed.

\section{Western blot analysis}

Cells and exosomes were lysed with RIPA lysis buffer containing protease and phosphatase inhibitors. Nuclear proteins were extracted with a kit (CWBIO, Beijing, China). Protein samples were separated by SDS-PAGE, and then transferred to PVDF membranes (Millipore, Burlington, MA, USA). After blocking for $1 \mathrm{~h}$, the PVDF membranes were incubated with primary antibodies overnight at $4^{\circ} \mathrm{C}$. The primary antibodies used were anti-CD81 (1 : 1,000; Cell Signaling Technology, Danvers, MA, USA),
anti-CD63 (1 : 1,000; Cell Signaling Technology), antiTSG-101 (1 : 1,000; Cell Signaling Technology), anti-calnexin (1 : 1,000; Cell Signaling Technology), anti-Galectin-3 (1 : 1,500; Abcam, Cambridge, UK), anti- $\alpha$-SMA ( 1 : 1,000; Bioworld Technology, Bloomington, MN, USA), anti-Collagen I (1 : 1,000; Bioworld Technology), anti-Periostin ( 1 : 1,000; Proteintech, Wuhan, China), anti-IL-1 $\beta$ (1 : 1,000; Bioworld Technology), anti-TNF- $\alpha$ (1 : 1,000; Bioworld Technology), anti-TGF- $\beta$ (1: 1,000; Abcam), anti- $\beta$-catenin ( $: 1,000$; Cell Signaling Technology), anti-GAPDH ( $: 3,000$; CWBIO, Beijing, China), and antiHistone (1:1,000; Cell Signaling Technology). The next day, membranes were incubated at $37^{\circ} \mathrm{C}$ for $1 \mathrm{~h}$ with horseradish peroxidase-linked goat anti-rabbit or anti-mouse antibodies (1 : 3,000; ABM, Richmond, Canada).

\section{Immunohistochemistry}

Myocardial tissues were fixed with $4 \%$ paraformaldehyde, embedded in a paraffin block, and cut into $3-\mu \mathrm{m}$ sections. After a series of deparaffinization and dehydration steps, endogenous peroxidase activity was blocked with $3 \% \mathrm{H}_{2} \mathrm{O}_{2}$, and then freshly prepared $0.01 \mathrm{~mol} / \mathrm{l}$ citrate buffer was used for antigen retrieval. After washing three times with PBS, the sections were blocked with 5\% BSA for $30 \mathrm{~min}$, and then incubated with the following primary antibodies: anti- $\alpha$-SMA (1 : 200; MAXIM, Fujian, China), anti-Periostin ( $: 200$; Proteintech), anti-Collagen I (1 : 200; Boster, Wuhan, China), anti-IL-1 $\beta$ (1 : 200; Bioworld Technology), anti-TNF- $\alpha$ (1:200; Bioworld Technology), and anti-TGF- $\beta$ ( $1: 300$; Abcam) overnight at $4^{\circ} \mathrm{C}$. Then the sections were incubated with biotin-labeled goat anti-mouse/rabbit secondary antibodies at $37^{\circ} \mathrm{C}$ for $30 \mathrm{~min}$. DAB chromogenic solution was used for color development, and hematoxylin was used for counter staining. Immunohistochemical images were captured with a Panoramic Scanner MIDI (3DHISTECH, Budapest, Hungary).

\section{Reverse transcription-polymerase chain reaction (RT-PCR)}

Fibroblasts were treated with LPS, LPS + hucMSC-ex, LPS + hucMSC-NC-ex, and LPS + hucMSC-si-ex. After 24 $\mathrm{h}$, the cells were collected and lysed with TRIzol reagent (Invitrogen) to extract total cellular RNA. Complementary DNA (cDNA) was obtained using a reverse transcription kit (CWBIO). RT-PCR was performed using UltraSYBR $\operatorname{mix}(\mathrm{CWBIO}) . \quad \beta$-actin was used as the endogenous reference gene for all RT-PCR experiments. PCR primers are listed in Table 1. 


\section{Immunofluorescence assay}

Immunofluorescence analysis of primary cardiac fibroblasts was performed as described previously (5). Anti- $\beta$ catenin primary antibody $(1: 300$; Cell Signaling Technology) was used. The secondary antibody was a fluorescently coupled antibody (1:1,000; Cell Signaling Technology). Nuclei were stained with Hoechst (Sigma-Aldrich). A fluorescence microscope (OLYMPUS DP73) was used to obtain images.

\section{Transwell migration assay}

The migration of cardiac fibroblasts was determined using Transwell chambers (Corning Inc., Corning, NY, USA). Primary fibroblasts $\left(1.5 \times 10^{5}\right.$ cells $\left./ \mathrm{ml}\right)$ were treated with LPS, LPS + hucMSC-ex, LPS + hucMSC-NC-ex, or LPS + hucMSC-si-ex for $24 \mathrm{~h}$, and then resuspended in serum-free $\alpha$-MEM. Next, $200 \mu 1$ of cell suspension was added to the upper chamber and $600 \mu 1$ of $\alpha$-MEM containing $10 \%$ FBS of was added to the lower chamber. After incubating in $5 \% \mathrm{CO}_{2}$ at $37^{\circ} \mathrm{C}$ for $8 \mathrm{~h}$, the cells in the upper chamber were removed. Meanwhile, cells adhered

Table 1. Primer sequences for the amplification of target genes

\begin{tabular}{|c|c|}
\hline Genes & Primer sequence $\left(5^{\prime}-3^{\prime}\right)$ \\
\hline \multirow[t]{2}{*}{$\beta$-actin } & Forward: 5'-TGTCACCAACTGGGACGATA-3' \\
\hline & Reverse: 5'-GGGGTGTTGAAGGTCTCA AA-3' \\
\hline \multirow[t]{2}{*}{ IL-1 $\beta$} & Forward: 5'-ATCTCACAGCAGCATCTCGACAAG-3' \\
\hline & Reverse: 5'-CACACTAGCAGGTCG TCATCATCC-3' \\
\hline \multirow[t]{2}{*}{ TNF- $\alpha$} & Forward: 5'-GCATGATCCGAGATGTGG AACTGG-3 \\
\hline & Reverse: 5'-CGCCACGAGCAGGAATGAGAAG-3' \\
\hline \multirow[t]{2}{*}{ TGF- $\beta$} & Forward: 5'-GGCACCATCCATGACATGAACCG-3' \\
\hline & Reverse: 5'-GCCGTACACAGCAGTTCTTCTCTG-3' \\
\hline \multirow[t]{2}{*}{ Galectin-3 } & Forward: 5'-GCCTTCCACTTTAACCCACG-3' \\
\hline & Reverse: 5'-AACCGACTGTCTTTCTTCCCTTC-3' \\
\hline
\end{tabular}

A

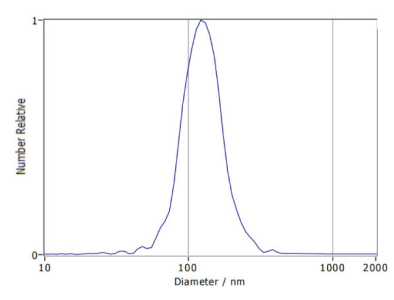

D

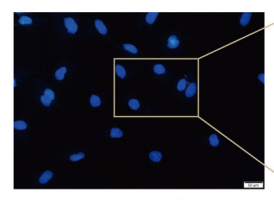

Control
B
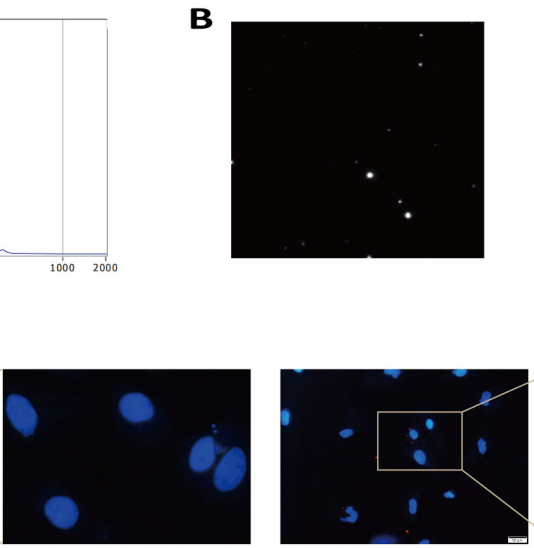

CM-Dil labeled-ex to the membrane of the lower chamber were fixed with $4 \%$ paraformaldehyde at room temperature for $30 \mathrm{~min}$, and then stained with $0.1 \%$ Crystal Violet. Images from three randomly selected fields were obtained with an inverted microscope (OLYMPUS DP73, Japan) and the cells were counted.

\section{Collagen gel contraction assay}

Cells were resuspended in serum-free $\alpha$-MEM to a final density of $2.5 \times 10^{5}$ cells $/ \mathrm{ml}$. Then $250 \mu 1$ of cell suspension was mixed with $200 \mu 1$ of Collagen I $(3 \mathrm{mg} / \mathrm{ml})$ (Gibco), $25 \mu 1$ of $10 \times$ DMEM (Gibco) and $25 \mu 1$ of 0.1 $\mathrm{mol} / \mathrm{l} \mathrm{NaOH}$. The mixture was spread in a 24-well plate $\left(500 \mu 1\right.$ per well) and incubated at $37^{\circ} \mathrm{C}$ for $1 \mathrm{~h}$. Then the gel was separated and cultured in 10\% FBS $\alpha$-MEM containing LPS, LPS + hucMSC-ex, LPS + hucMSC-NC-ex, or LPS + hucMSC-si-ex. The control group was cultured in $10 \%$ FBS $\alpha$-MEM only. After $24 \mathrm{~h}$, the gel contraction images were taken with a camera, and the surface area was measured using Image J software to quantify the degree of contraction.

\section{Statistical analysis}

All data were processed with Prism 5.0 software and are expressed as the mean \pm SD. Student's $t$-test or one-way ANOVA was used to compare experimental groups and the relative control group. $\mathrm{p}$ values $<0.05$ were considered significant.

\section{Results}

\section{Characterization of HucMSC-ex}

Exosomes are tiny vesicles secreted by most cells. We measured the particle size of hucMSC-ex and recorded motion images by NTA. The particle size of hucMSC-ex
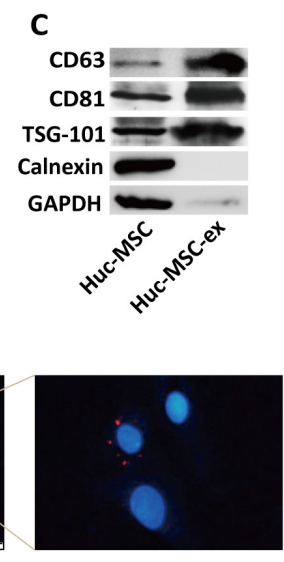

Fig. 1. Characterization of hucMSC-ex. (A) The particle sizes of hucMSC-ex. (B) Image of hucMSC-ex. (C) Exosomal marker proteins CD63, CD81, and TSG-101 and the negative control calnexin were detected by western blot. (D) The internalization of exosomes by fibroblasts. Exosomes were stained with CM-Dil (red) and nuclei were stained with Hoechst (blue). Scale bar $=50 \mu \mathrm{m}$. 
was $30 \sim 150 \mathrm{~nm}$ (Fig. 1A and 1B). Additionally, western blot results showed that the exosomal marker proteins CD63, CD81, and TSG-101 were found in hucMSC-ex, but calnexin and GAPDH were not found in hucMSC-ex (Fig. 1C). Exosomes can be taken up by many kinds of cells. We labeled hucMSC-ex with CM-Dil and co-cultured them with cardiac fibroblasts for $12 \mathrm{~h}$. The results showed that the labeled exosomes were distributed around the nuclei of cardiac fibroblasts (Fig. 1D). These results demonstrated that hucMSC-ex entered into cardiac fibroblasts, which provided a strong basis for subsequent experiments.

\section{HucMSC-ex promoted fibroblast-to-myofibroblast differentiation in inflammatory environment}

Next, primary fibroblasts were treated with LPS or LPS+ hucMSC-ex for $24 \mathrm{~h}$. Western blots showed that $\alpha$-SMA, Collagen I, and Periostin expression were all up-regulated in the LPS + ex group compared with the LPS group (Fig. $2 \mathrm{~A}$ and $2 \mathrm{~B}$ ). Additionally, the collagen contraction ability of cells in the LPS + ex group was enhanced compared with LPS-treated cells, but their migration ability was weakened (Fig. 2C and 2D).

\section{HucMSC-ex-Galectin-3 promoted the differentiation of} cardiac fibroblasts into myofibroblasts

Thus, hucMSC-ex can promote fibroblast-to-myofibroblast differentiation during inflammatory environment (5). Previous studies have shown that Galectin-3 also promotes the differentiation of fibroblasts into myofibroblasts during inflammation $(10,12)$.

Thus, we next investigated if the effects of hucMSC-ex involved Galectin-3. We detected the expression of Galectin-3 protein in hucMSCs and hucMSC-ex by western blot. The results illustrated that hucMSCs and hucMSC-ex contained Galectin-3 (Fig. 3A). Then, to understand the biological role of Galectin-3 in hucMSC-ex, Galectin-3 expression in hucMSCs was silenced by siRNA. As shown in Fig. 3B and 3C, Galectin-3 expression was the lowest in hucMSCs with Galectin-3 siRNA1 compared with the other groups. Similarly, Galectin-3 was knocked down in hucMSC-ex with a Galectin-3 siRNAl (Fig. 3D). The results showed that silencing Galectin-3 in hucMSCs decreased Galectin-3 levels in hucMSC-ex. Next, we assessed the possible role of Galectin-3 in promoting the differentiation of cardiac fibroblasts into myofibroblasts. Primary fibroblasts were treated with LPS, LPS + hucMSC-ex, LPS + hucMSC-NC-ex, or LPS + hucMSC-
A

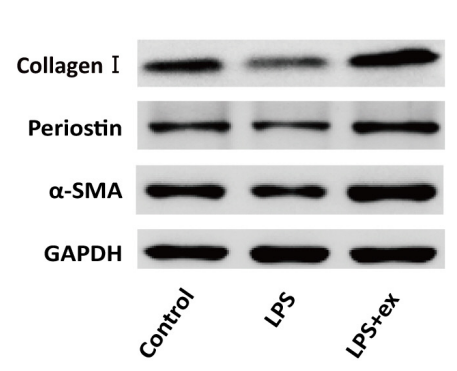

C

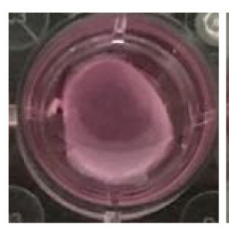

Control

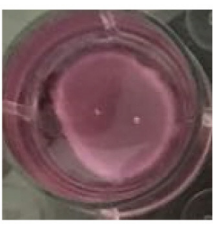

LPS

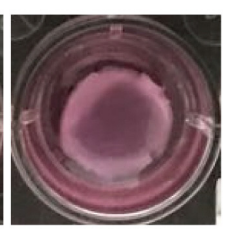

LPS+ex

D

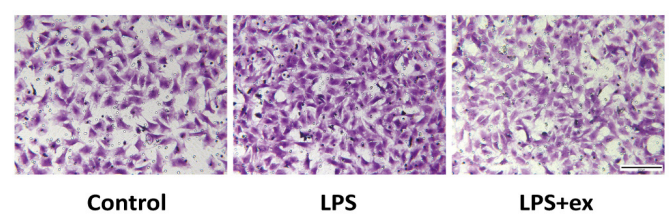

B

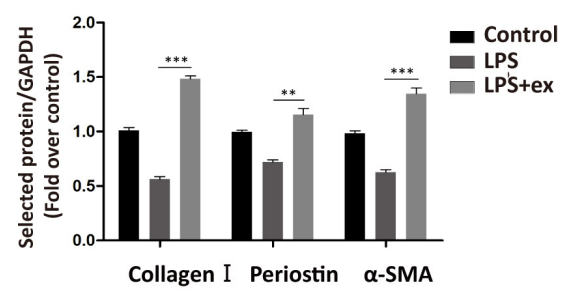

Fig. 2. HucMSC-ex promoted fibroblast-to-myofibroblast differentiation in inflammatory environments. Cardiac fibroblasts were treated with LPS (100 $\mathrm{ng} / \mathrm{ml})$ or LPS+hucMSCex $(200 \mu \mathrm{g} / \mathrm{ml})$ for $24 \mathrm{~h}$; the control group did not receive any treatment. (A, B) Collagen I, Periostin, and $\alpha$-SMA expression were detected by western blot. (C) Collagen contraction analysis was used to determine the collagen contractility of cardiac fibroblasts treated with LPS $(100 \mathrm{ng} / \mathrm{ml})$ or LPS+ hucMSC-ex $(200 \mu \mathrm{g} / \mathrm{ml})$ for $24 \mathrm{~h}$. (D) Cardiac fibroblasts were treated with LPS $(100 \mathrm{ng} / \mathrm{ml})$ or LPS + hucMSCex $(200 \mu \mathrm{g} / \mathrm{ml})$ for $24 \mathrm{~h}$, Transwell migration assays were used to detect the migration ability of cardiac fibroblasts. Scale bar $=100 \mu \mathrm{m} ;{ }^{*} \mathrm{p}<$ $0.05, * * p<0.01$, and $* * * p<0.001$. 
si-ex for $24 \mathrm{~h}$ in vitro. Western blot was used to detect $\alpha$ SMA, Collagen I and Periostin expression. The expression of these proteins was increased in the hucMSC-ex group, while it was decreased in hucMSC-si-ex group (Fig. 4A). Immunohistochemistry was used to detect the expression of $\alpha$-SMA, Collagen I and Periostin, which were increased in the AMI+ ex group compared with the AMI+ PBS group; when Galectin-3 siRNA was used, the expression was reduced compared with the group treated with NC sequence (Fig. 4B). In conclusion, in an in- flammatory environment, hucMSC-ex-Galectin-3 promoted the differentiation of cardiac fibroblasts into myofibroblasts in vivo and in vitro. The differentiation of fibroblasts into myofibroblasts increased the contraction ability and weakened the migration ability. The contractility of fibroblasts was weakened in the LPS + hucMSC-si-ex group compared with the LPS + hucMSC-NC-ex group. In contrast, their migration ability showed the opposite trend (Fig. 5A and 5B).
A

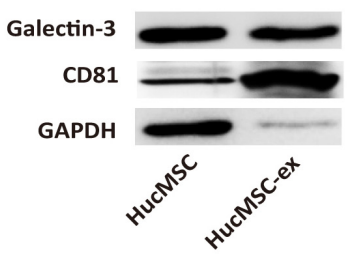

C

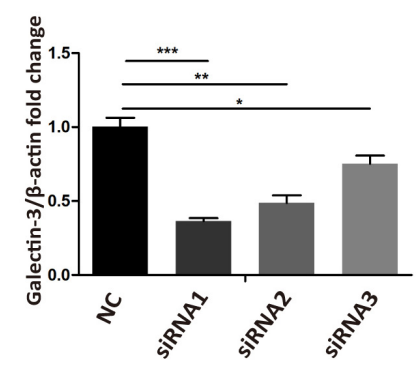

B

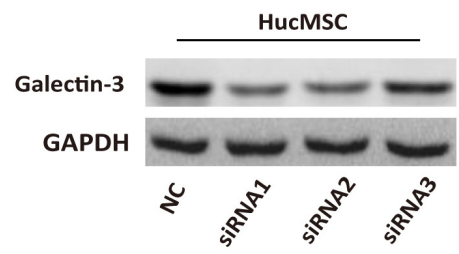

D

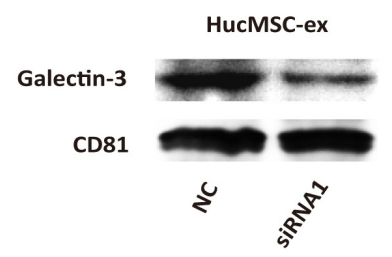

Fig. 3. SiRNA-mediated knockdown of hucMSC-Galectin-3. (A) Galectin-3 protein expression in hucMSC and hucMSC-ex were detected by western blot. (B, C) HucMSCs were transfected with Galectin-3 siRNA, and then Galectin-3 protein and mRNA expression were detected by western blot and qRT-PCR, respectively. (D) Levels of Galectin-3 protein in hucMSC-ex following Galectin-3 knockdown were detected by western blot. ${ }^{*} \mathrm{p}<0.05,{ }^{* *} \mathrm{p}<0.01$, and $* * * p<0.001$.

\section{A}

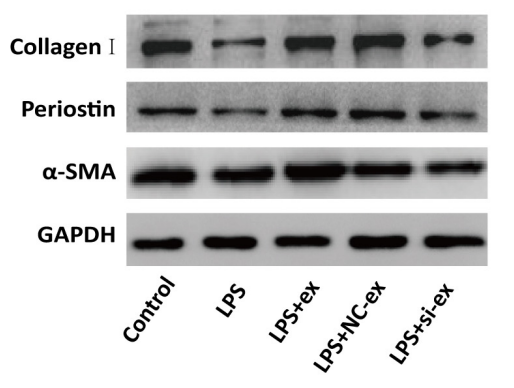

B
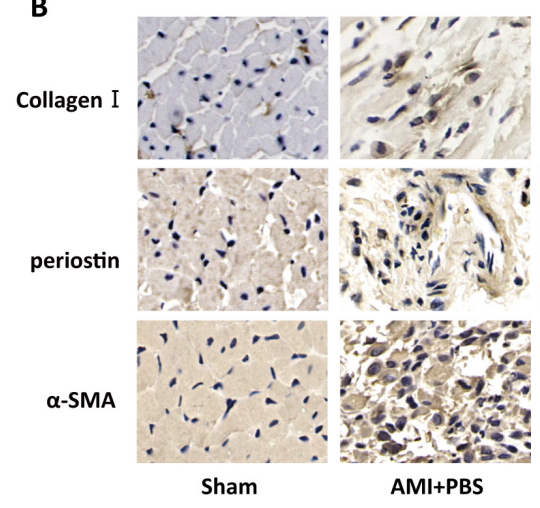

AMI+PBS
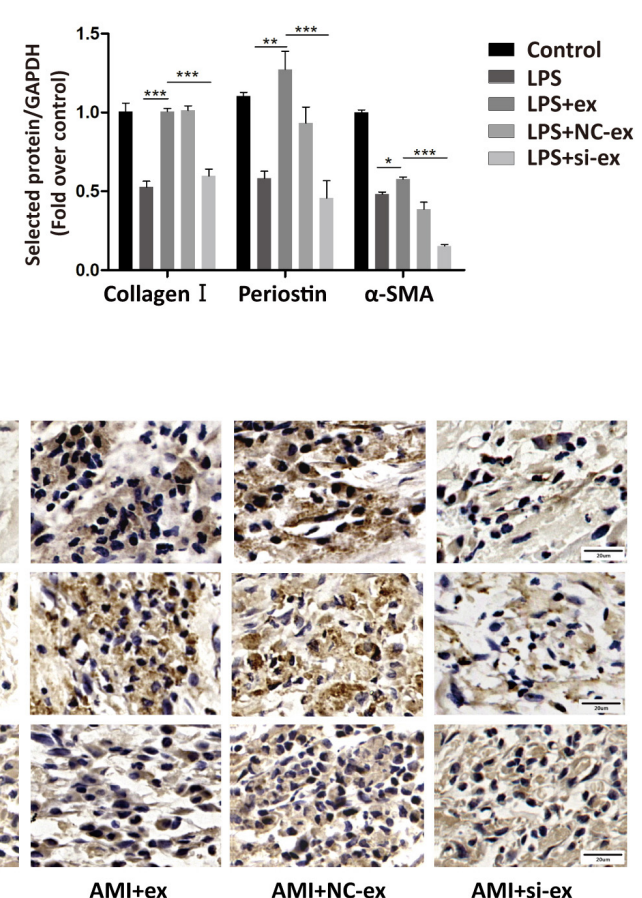

Fig. 4. HucMSC-ex-Galectin-3 promoted the differentiation of cardiac fibroblasts into myofibroblasts. Cardiac fibroblasts were treated with LPS (100 ng/ml), LPS + hucMSC-ex (200 $\mu \mathrm{g} / \mathrm{ml})$, LPS + hucMSC-NC-ex (200 $\mu \mathrm{g} / \mathrm{ml})$, or LPS + hucMSC-si-ex (200 $\mu \mathrm{g} / \mathrm{ml}$ ) for $24 \mathrm{~h}$. (A) Collagen I, Periostin, and $\alpha$-SMA levels were detected by western blot. (B) Two days after myocardial infarction was induced in SD rats, Collagen I, Periostin, and $\alpha$-SMA expression in the infarcted area were detected by immunohistochemistry. Scale bar= $20 \mu \mathrm{m} .{ }^{*} \mathrm{p}<0.05, * * \mathrm{p}<0.01$, and $* * * \mathrm{p}<0.001$. 
A

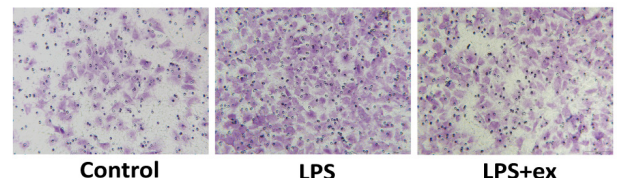

$$
\text { Control }
$$
LPS LPS+ex

B

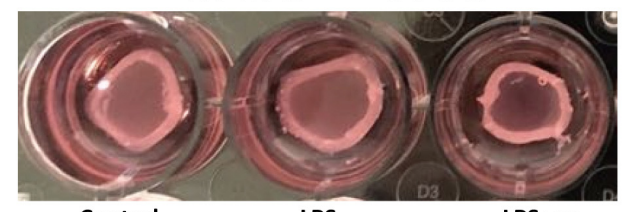

Control LPS LPS+ex

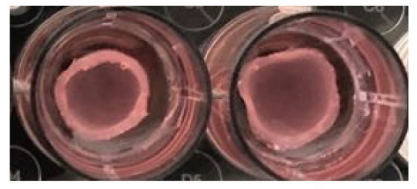

LPS+NC-ex
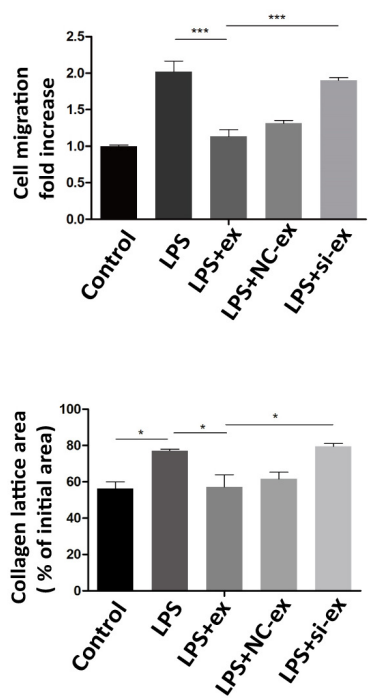

Fig. 5. HucMSC-ex-Galectin-3 altered the function of cardiac fibroblasts. Cardiac fibroblasts were treated with LPS $(100 \mathrm{ng} / \mathrm{ml})$, LPS + hucMSC-ex (200 $\mu \mathrm{g} / \mathrm{ml})$, LPS+hucMSC-NC-ex (200 $\mu \mathrm{g} / \mathrm{ml})$, or LPS+hucMSC-si-ex (200 $\mu \mathrm{g} / \mathrm{ml}$ ) for $24 \mathrm{~h}$. (A) Transwell migration assays were used to detect the migration ability of cardiac fibroblasts. Scale bar $=100 \mu \mathrm{m}$. (B) CoIlagen contraction analysis detected the collagen contractility of cardiac fibroblasts. ${ }^{*} \mathrm{p}<0.05$ and ${ }^{* * *} \mathrm{p}<0.001$.
A

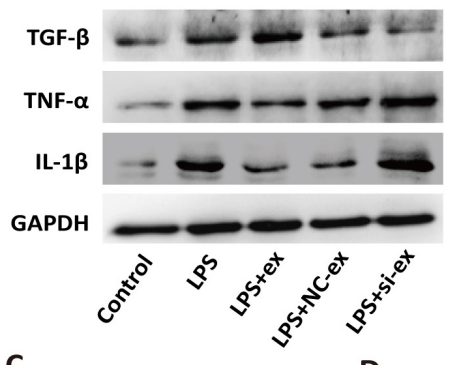

C

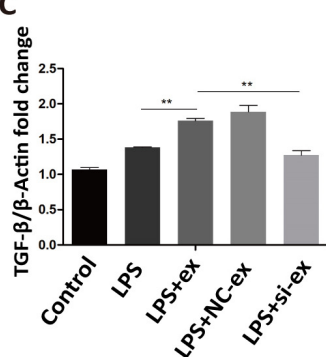

D

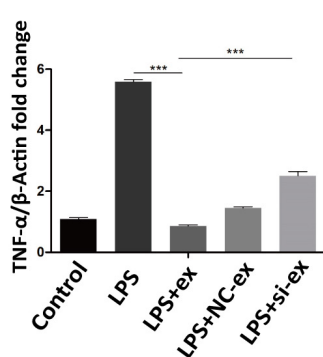

B

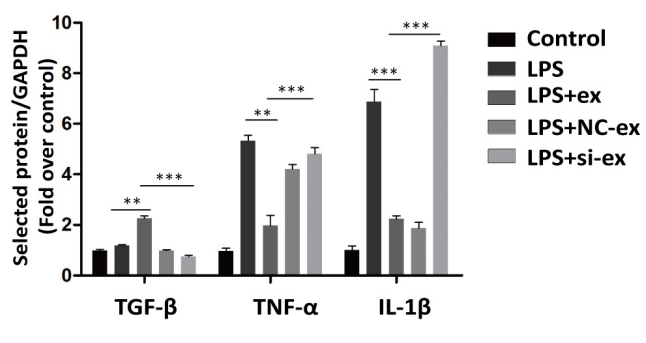

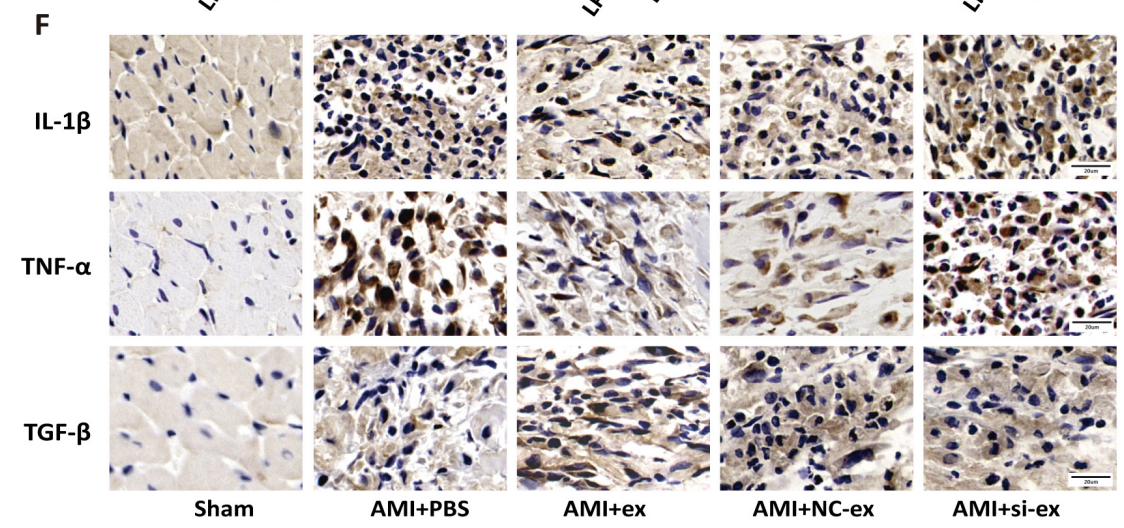

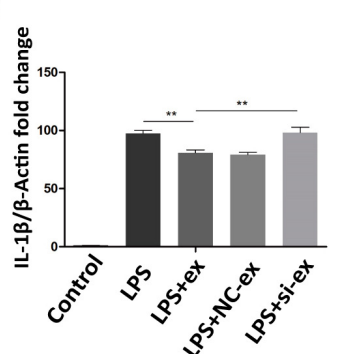

Fig. 6. HucMSC-ex-Galectin-3 promoted the transformation of fibroblasts to an anti-inflammatory phenotype. Cardiac fibroblasts were treated with LPS (100 ng/ml), LPS+hucMSCex $(200 \mu \mathrm{g} / \mathrm{ml})$, LPS +hucMSC-NC-ex (200 $\mu \mathrm{g} / \mathrm{ml})$, or LPS+hucMSC-si-ex (200 $\mu \mathrm{g} / \mathrm{ml})$ for $24 \mathrm{~h}$. (A, B) IL-1 $\beta$, TNF- $\alpha$, and TGF- $\beta$ protein levels were detected by western blot. $(\mathrm{C} \sim$ E) The mRNA expression of IL-1 $\beta$, TNF- $\alpha$, and TGF- $\beta$ were detected by qRT-PCR. (F) Two days after myocardial infarction was induced, IL-1 $\beta$, TNF- $\alpha$, and TGF- $\beta$ expression in the infarcted area were detected by immunohistochemistry. Scale bar = $20 \mu \mathrm{m} .{ }^{*} \mathrm{p}<0.05, * * \mathrm{p}<0.01$, and $* * * \mathrm{p}<0.001$. 
HucMSC-ex-Galectin-3 promoted the transformation of fibroblasts to an anti-inflammatory phenotype

It has been reported that myofibroblasts have anti-inflammatory effects (17-19). Primary fibroblasts were treated with LPS, LPS + hucMSC-ex, LPS + hucMSC-NC-ex, or LPS + hucMSC-si-ex for $24 \mathrm{~h}$. RT-PCR and western blot were used to detect the mRNA and protein levels of IL- $\beta$, TNF- $\alpha$, and TGF- $\beta$. In the hucMSC-si-ex group, IL-1 $\beta$ and TNF- $\alpha$ expression were increased and TGF$\beta$ expression was reduced compared with the hucMSC-ex group (Fig. 6A $\sim$ E). Therefore, hucMSC-ex-Galectin-3 promoted the conversion of fibroblasts to an anti-inflammatory phenotype. In vivo, immunohistochemistry was used to detect the expression of inflammatory factors. In the hucMSC-si-ex group, TGF- $\beta$ expression was decreased, while IL-1 $\beta$ and TNF- $\alpha$ were upregulated (Fig. $6 \mathrm{~F})$.

\section{HucMSC-ex-Galectin-3-mediated} fibroblast-to-myofibroblast differentiation was associated with increased $\beta$-catenin expression

It has been reported that Galectin-3 can stabilize $\beta$ - catenin through a series of steps (7). Therefore, we hypothesized that Galectin-3 derived from hucMSC-ex could modulate cardiac fibroblasts by stabilizing $\beta$-catenin in an inflammatory environment. After primary fibroblasts were treated with LPS +hucMSC-ex for $24 \mathrm{~h}, \beta$-catenin expression was increased in both the cytoplasm and nucleus compared with LPS-only treatment. When treated with LPS + si-ex, the opposite results were found (Fig. 7A $\sim$ C). To further determine whether hucMSC-ex-Galectin-3-induced fibroblast-to-myofibroblast transformation depended on $\beta$ catenin, ICG-001 has been used. ICG-001, a small molecule inhibitor, was modified onto CREB-binding protein (CBP) to block the interaction between CBP and $\beta$-catenin, which inhibited transcription of target genes (20). When ICG-001 was used, $\beta$-catenin expression in fibroblasts decreased in a concentration-dependent manner (Fig. 7D). Furthermore, decreased $\alpha$-SMA, Collagen I, and Periostin expression was found after treatment with the effective inhibitor concentration $(5 \mu \mathrm{M})$ (Fig. 7E).
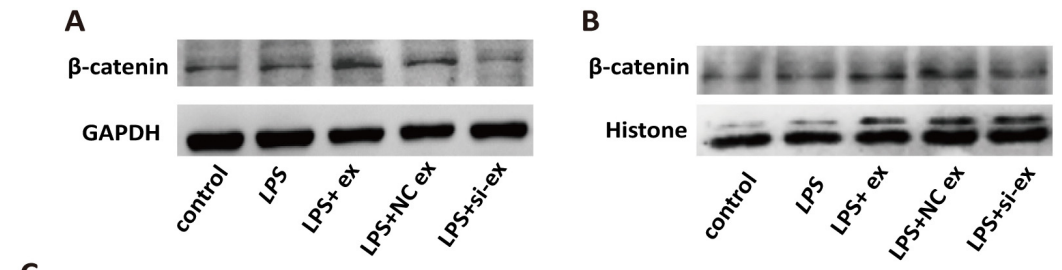

C

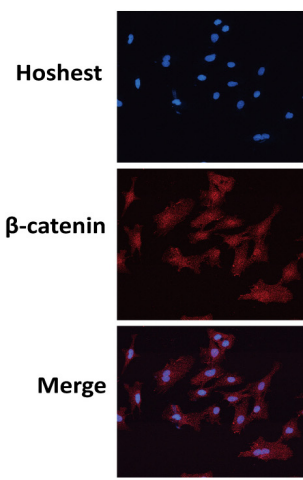

Control

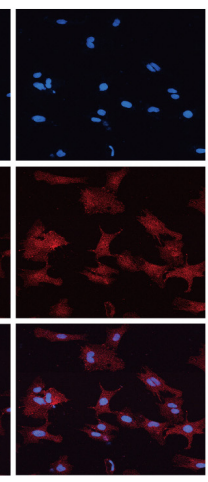

LPS

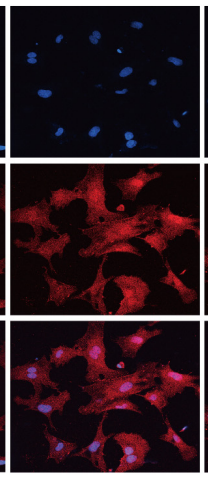

LPS+ex

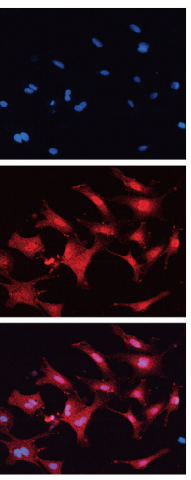

LPS+NC-ex

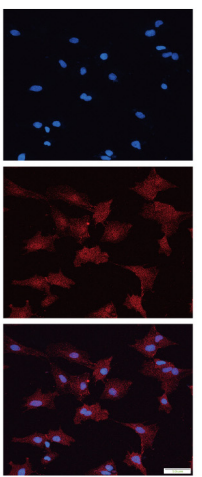

LPS+si-ex

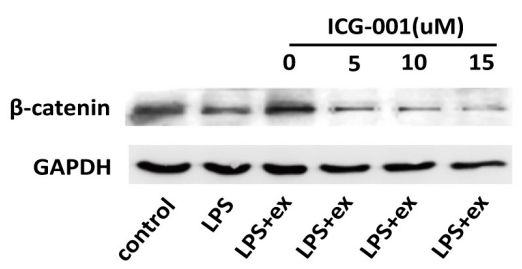

E

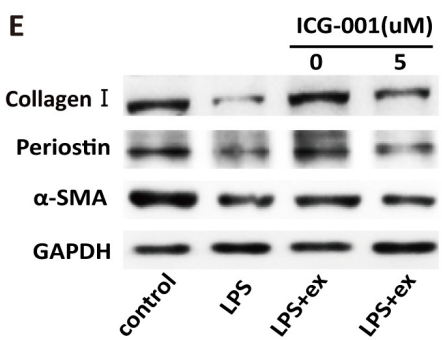

Fig. 7. HucMSC-ex-Galectin-3-induced fibroblast-to-myofibroblast transformation was associated with increased $\beta$-catenin expression. Cardiac fibroblasts were treated with LPS (100 $\mathrm{ng} / \mathrm{ml}), \mathrm{LPS}+$ hucMSC-ex $(200 \mu \mathrm{g} / \mathrm{ml})$, LPS+hucMSC-NC-ex $(200 \mu \mathrm{g} / \mathrm{ml})$, or LPS + hucMSC-si-ex $(200 \mu \mathrm{g} / \mathrm{ml})$ for $24 \mathrm{~h}$. (A) $\beta$-catenin expression was detected by western blot. (B) Nuclear $\beta$-catenin levels were also measured by western blot. (C) $\beta$-catenin expression as detected by immunofluorescence. Scale bar $=100 \mu \mathrm{m}$. (D) Cardiac fibroblasts were treated with LPS (100 ng/ml), LPS + hucMSCex $(200 \mu \mathrm{g} / \mathrm{ml})$, LPS+hucMSC-ex (200 $\mu \mathrm{g} / \mathrm{ml})+\mathrm{ICG}-001(0,5,10,15 \mu \mathrm{M})$ for $24 \mathrm{~h}$, and then $\beta$-catenin levels were detected by western blot. (E) Cardiac fibroblasts were treated with LPS (100 ng/ml), LPS + hucMSC-ex (200 $\mu \mathrm{g} / \mathrm{ml})$, LPS + hucMSC-ex (200 $\mu \mathrm{g} / \mathrm{ml})+\mathrm{ICG}-001(5 \mu \mathrm{M})$ for $24 \mathrm{~h}$, and then Collagen I, Periostin, and $\alpha$-SMA expression were detected by western blot. 


\section{Discussion}

The ability of bone marrow mesenchymal stem cell exosomes to repair myocardial injury has been studied by many researchers. The primary mechanisms of this activity involve reducing apoptosis of myocardial cells, regulating macrophage activation to reduce inflammation, and promoting angiogenesis (21-23). In recent years, hucMSC-ex has been considered a new method of facilitating tissue repair and regeneration. The protective effects of hucMSCex on myocardial injury mostly focus on reducing the apoptosis of myocardial cells to improve myocardial function $(24,25)$. Our previous studies have also shown that hucMSC-ex could improve the repair of myocardial injury, attenuate proinflammatory factor secretion, and inhibit myocardial apoptosis by promoting the differentiation of cardiac fibroblasts into myofibroblasts in an inflammatory environment. As previously discussed, the differentiation of fibroblasts into myofibroblasts is a key step in the repair of myocardial injury. In the inflammatory phase of myocardial damage, myocardial fibroblasts are activated to promote inflammation $(26,27)$. To maintain myocardial structure, in the proliferation stage, myocardial fibroblasts differentiate into myofibroblasts, which can secrete large amounts of extracellular matrix and inhibit inflammation (18). In the inflammatory phase, promoting the differentiation of cardiac fibroblasts into myofibroblasts could mitigate the inflammatory response, weaken the degradation of extracellular matrix, reduce the apoptosis of cardiomyocytes, and better maintain the original structure of the myocardium. Immediately repairing damage after myocardial injury may reduce the risk of myocardial fibrosis and heart failure.

The purpose of this study was to determine the key proteins in hucMSC-ex that promoted the differentiation of myocardial fibroblasts into myofibroblasts in an inflammatory environment and to explore the underlying mechanism. Galectin-3 has been reported to play an active role in injury repair following myocardial infarction. The study by Sharma et al. (28) showed that Galectin-3 could transform stationary fibroblasts into myofibroblasts in vitro. In vivo, Galectin-3 plays an important role in cardiac fibrosis and remodeling. We have also confirmed that hucMSC- ex contain Galectin-3 protein. We investigated the effect of hucMSC-ex-derived Galectin-3 on the differentiation of fibroblasts into myofibroblasts in vitro and in vivo. The results showed that hucMSC-ex-Galectin-3 promoted the expression of proteins related to fibroblast-to-myofibroblast differentiation, and stimulated the transformation of fibroblasts to an anti-inflammatory phenotype.

The interaction between $\beta$-catenin and Galectin- 3 has been reported in previous studies $(29,30)$. Galectin- 3 can bind to $\beta$-catenin and directly enter the nucleus to regulate the transcription of target genes (15). In an inflammatory environment, cardiac fibroblasts were treated with hucMSC-ex for $24 \mathrm{~h}$, and the levels of $\beta$-catenin in total protein and nucleoprotein were up-regulated. This phenomenon was reversed in the hucMSC-si-ex group. Combined with the results of Fig. 4A, we speculate that myofibroblast activation may be related to $\beta$-catenin expression. ICG-001 is a $\beta$-catenin transcriptional inhibitor, which inhibited the ability of $\beta$-catenin to regulate transcription. Cardiac fibroblasts were treated with LPS + hucMSC-ex+ICG-001 for $24 \mathrm{~h}$, compared with the hucMSC-ex group, expression of the myofibroblast activation-related proteins $\alpha$-SMA, Collagen I, and Periostin were significantly downregulated. These results showed that hucMSC-ex-Galectin-3 promoted myocardial fibroblast-to-myofibroblast differentiation and increased $\beta$ -catenin expression. In the study by Mackinnon et al. (31), Galectin-3 extracted from rat alveolar epithelial cells stimulated with TGF- $\beta 1$ increased $\beta$-catenin activation by inhibiting GSK-3 $\beta$ activity. In primary alveolar epithelial cells, nuclear translocation of $\beta$-catenin was inhibited by treatment with a Galectin-3 inhibitor, which attenuated pulmonary fibrosis in vivo (31). These results showed that Galectin-3 could promote $\beta$-catenin nuclear translocation, which was consistent with our results.

Mesenchymal stem cell therapy inevitably faces challenges such as low survival rate and short resident time. Mesenchymal stem cell-derived exosomes are suitable cell substitutes. In our previous research, we have determined that hucMSC-ex promotes the differentiation of cardiac fibroblasts into myofibroblasts, which has cardioprotective effects in an inflammatory environment. In this study, we found the key protein in hucMSC-ex that promotes fibroblast-to-myofibroblast differentiation. Modulating the expression of key proteins in exosomes could be a method of improving treatments for myocardial injury.

In conclusion, we found that Galectin-3 in hucMSC-ex promoted the differentiation of cardiac fibroblasts into myofibroblasts in an inflammatory environment, which was associated with increased $\beta$-catenin levels in fibroblasts. This ability of hucMSC-ex may provide a new therapeutic strategy for myocardial injury.

\section{Acknowledgments}

This work was supported by the National Natural Science Foundation of China [grant number 81800270] 
and the Zhenjiang Municipal Social Development Project [grant number SH2018039].

\section{Potential Conflict of Interest}

The authors have no conflicting financial interest.

\section{References}

1. Fan D, Takawale A, Lee J, Kassiri Z. Cardiac fibroblasts, fibrosis and extracellular matrix remodeling in heart disease. Fibrogenesis Tissue Repair 2012;5:15

2. Tallquist MD, Molkentin JD. Redefining the identity of cardiac fibroblasts. Nat Rev Cardiol 2017;14:484-491

3. Shin EY, Wang L, Zemskova M, Deppen J, Xu K, Strobel F, García AJ, Tirouvanziam R, Levit RD. Adenosine production by biomaterial-supported mesenchymal stromal cells reduces the innate inflammatory response in myocardial ischemia/reperfusion injury. J Am Heart Assoc 2018;7:e006949

4. Teng X, Chen L, Chen W, Yang J, Yang Z, Shen Z. Mesenchymal stem cell-derived exosomes improve the microenvironment of infarcted myocardium contributing to angiogenesis and anti-inflammation. Cell Physiol Biochem 2015;37:2415-2424

5. Shi Y, Yang Y, Guo Q, Gao Q, Ding Y, Wang H, Xu W, Yu B, Wang M, Zhao Y, Zhu W. Exosomes derived from human umbilical cord mesenchymal stem cells promote fibroblast-to-myofibroblast differentiation in inflammatory environments and benefit cardioprotective effects. Stem Cells Dev 2019;28:799-811

6. Deng H, Sun C, Sun Y, Li H, Yang L, Wu D, Gao Q, Jiang $\mathrm{X}$. Lipid, protein, and microRNA composition within mesenchymal stem cell-derived exosomes. Cell Reprogram 2018;20:178-186

7. $\mathrm{Hu} \mathrm{K}, \mathrm{Gu} \mathrm{Y}$, Lou L, Liu L, Hu Y, Wang B, Luo Y, Shi J, Yu X, Huang H. Galectin-3 mediates bone marrow microenvironment-induced drug resistance in acute leukemia cells via Wnt/ $\beta$-catenin signaling pathway. J Hematol Oncol 2015;8:1

8. de Boer RA, Voors AA, Muntendam P, van Gilst WH, van Veldhuisen DJ. Galectin-3: a novel mediator of heart failure development and progression. Eur J Heart Fail 2009;11: 811-817

9. Li LC, Li J, Gao J. Functions of galectin-3 and its role in fibrotic diseases. J Pharmacol Exp Ther 2014;351:336-343

10. Meijers WC, van der Velde AR, Pascual-Figal DA, de Boer RA. Galectin-3 and post-myocardial infarction cardiac remodeling. Eur J Pharmacol 2015;763(Pt A):115-121

11. González GE, Cassaglia P, Noli Truant S, Fernández MM, Wilensky L, Volberg V, Malchiodi EL, Morales C, Gelpi RJ. Galectin-3 is essential for early wound healing and ventricular remodeling after myocardial infarction in mice. Int J Cardiol 2014;176:1423-1425

12. Li M, Yuan Y, Guo K, Lao Y, Huang X, Feng L. Value of Galectin-3 in acute myocardial infarction. Am J Cardiovasc
Drugs 2020;20:333-342

13. Zhao X, Hua Y, Chen H, Yang H, Zhang T, Huang G, Fan H, Tan Z, Huang X, Liu B, Zhou Y. Aldehyde dehydrogenase-2 protects against myocardial infarction-related cardiac fibrosis through modulation of the Wnt/ $\beta$-catenin signaling pathway. Ther Clin Risk Manag 2015;11:1371-1381

14. Ge Z, Li B, Zhou X, Yang Y, Zhang J. Basic fibroblast growth factor activates $\beta$-catenin/RhoA signaling in pulmonary fibroblasts with chronic obstructive pulmonary disease in rats. Mol Cell Biochem 2016;423:165-174

15. Shimura T, Takenaka Y, Tsutsumi S, Hogan V, Kikuchi A, Raz A. Galectin-3, a novel binding partner of betacatenin. Cancer Res 2004;64:6363-6367

16. Qiao C, Xu W, Zhu W, Hu J, Qian H, Yin Q, Jiang R, Yan Y, Mao F, Yang H, Wang X, Chen Y. Human mesenchymal stem cells isolated from the umbilical cord. Cell Biol Int 2008;32:8-15

17. Ma Y, Iyer RP, Jung M, Czubryt MP, Lindsey ML. Cardiac fibroblast activation post-myocardial infarction: current knowledge gaps. Trends Pharmacol Sci 2017;38:448-458

18. Nagpal V, Rai R, Place AT, Murphy SB, Verma SK, Ghosh AK, Vaughan DE. MiR-125b is critical for fibroblast-to-myofibroblast transition and cardiac fibrosis. Circulation 2016; 133:291-301

19. Nakaya $M$, Watari K, Tajima $M$, Nakaya T, Matsuda $S$, Ohara H, Nishihara H, Yamaguchi $H$, Hashimoto A, Nishida $M$, Nagasaka A, Horii $Y$, Ono $H$, Iribe $G$, Inoue $\mathrm{R}$, Tsuda $M$, Inoue $\mathrm{K}$, Tanaka $\mathrm{A}$, Kuroda $M$, Nagata $S$, Kurose H. Cardiac myofibroblast engulfment of dead cells facilitates recovery after myocardial infarction. J Clin Invest 2017;127:383-401

20. Liu Y, Chen H, Zheng P, Zheng Y, Luo Q, Xie G, Ma Y, Shen L. ICG-001 suppresses growth of gastric cancer cells and reduces chemoresistance of cancer stem cell-like population. J Exp Clin Cancer Res 2017;36:125

21. Sun XH, Wang X, Zhang Y, Hui J. Exosomes of bone-marrow stromal cells inhibit cardiomyocyte apoptosis under ischemic and hypoxic conditions via miR-486-5p targeting the PTEN/PI3K/AKT signaling pathway. Thromb Res 2019; 177:23-32

22. Xu R, Zhang F, Chai R, Zhou W, Hu M, Liu B, Chen X, Liu M, Xu Q, Liu N, Liu S. Exosomes derived from pro-inflammatory bone marrow-derived mesenchymal stem cells reduce inflammation and myocardial injury via mediating macrophage polarization. J Cell Mol Med 2019;23:76177631

23. Sun J, Shen H, Shao L, Teng X, Chen Y, Liu X, Yang Z, Shen Z. HIF-1 $\alpha$ overexpression in mesenchymal stem cellderived exosomes mediates cardioprotection in myocardial infarction by enhanced angiogenesis. Stem Cell Res Ther 2020;11:373

24. Gu X, Li Y, Chen K, Wang X, Wang Z, Lian H, Lin Y, Rong X, Chu M, Lin J, Guo X. Exosomes derived from umbilical cord mesenchymal stem cells alleviate viral myocarditis through activating $\mathrm{AMPK} / \mathrm{mTOR}$-mediated autophagy flux pathway. J Cell Mol Med 2020;24:7515-7530 
25. Wang XL, Zhao YY, Sun L, Shi Y, Li ZQ, Zhao XD, Xu CG, Ji HG, Wang M, Xu WR, Zhu W. Exosomes derived from human umbilical cord mesenchymal stem cells improve myocardial repair via upregulation of Smad7. Int J Mol Med 2018;41:3063-3072

26. Sandanger $\varnothing$, Ranheim T, Vinge LE, Bliksøen $M$, Alfsnes K, Finsen AV, Dahl CP, Askevold ET, Florholmen G, Christensen G, Fitzgerald KA, Lien E, Valen G, Espevik T, Aukrust P, Yndestad A. The NLRP3 inflammasome is up-regulated in cardiac fibroblasts and mediates myocardial ischaemia-reperfusion injury. Cardiovasc Res 2013;99: 164-174

27. Shinde AV, Frangogiannis NG. Fibroblasts in myocardial infarction: a role in inflammation and repair. J Mol Cell Cardiol 2014;70:74-82

28. Sharma UC, Pokharel S, van Brakel TJ, van Berlo JH, Cleutjens JP, Schroen B, André S, Crijns HJ, Gabius HJ,
Maessen J, Pinto YM. Galectin-3 marks activated macrophages in failure-prone hypertrophied hearts and contributes to cardiac dysfunction. Circulation 2004;110:3121-3128 29. Liu Y, Xie L, Wang D, Li D, Xu G, Wang L, Zhou H, $\mathrm{Yu} \mathrm{Y}$, Lin Z, Lu H. Galectin-3 and $\beta$-catenin are associated with a poor prognosis in serous epithelial ovarian cancer. Cancer Manag Res 2018;10:3963-3971

30. Song M, Pan Q, Yang J, He J, Zeng J, Cheng S, Huang Y, Zhou ZQ, Zhu Q, Yang C, Han Y, Tang Y, Chen H, Weng DS, Xia JC. Galectin-3 favours tumour metastasis via the activation of $\beta$-catenin signalling in hepatocellular carcinoma. Br J Cancer 2020;123:1521-1534

31. Mackinnon AC, Gibbons MA, Farnworth SL, Leffler H, Nilsson UJ, Delaine T, Simpson AJ, Forbes SJ, Hirani N, Gauldie J, Sethi T. Regulation of transforming growth factor- $\beta 1$-driven lung fibrosis by galectin-3. Am J Respir Crit Care Med 2012;185:537-546 